\title{
A influência da avaliação baseada em desempenho no sistema de valorização do mérito: uma proposta para as escolas militares de formação e aperfeiçoamento
}

Este trabalho demonstra a necessidade de realizar uma avaliação padronizada, baseada no desempenho do aluno para possibilitar a seleção de militares de diferentes turmas de formação e aperfeiçoamento. O propósito deste estudo é encontrar uma fórmula baseada na média e no desvio padrão, capaz de padronizar as notas dos alunos e possibilitar a comparação entre turmas diferentes. Este intento será alcançado mediante pesquisa bibliográfica do assunto, comparativo com outras instituições que já utilizam a nota padronizada, além da pesquisa do desvio padrão e da média entre turmas de aperfeiçoamento da Escola de Aperfeiçoamento de Oficiais. Foi possível evidenciar que a nota padronizada, atualmente, é a forma mais coerente de comparação de turmas e provas diferentes, contribuindo para uma análise de desempenho mais confiável e baseada no desempenho. Observou-se distorções nas notas e médias das turmas da Escola de Aperfeiçoamento de Oficiais próximas, nas quais os militares concorrem aos processos seletivos com notas não padronizadas, demonstrando uma discrepância do sistema atual que utiliza a nota bruta. Tal fato possibilitou a concepção de uma fórmula capaz de padronizar as notas e evitar as distorções nos processos seletivos.

Palavras-chave: Escolas Militares; Mérito; Desempenho; Avaliação.

\section{The influence of performance-based evaluation on the merit valuation system: a proposal for military training schools}

This paper demonstrates the need to perform a standardized assessment based on student performance to enable the selection of military personnel from different training and further training groups. The purpose of this study is to find a formula based on the mean and standard deviation that can standardize students' grades and enable comparison between different classes. This intent will be achieved through a bibliographic research of the subject, comparative with other institutions that already use the standardized grade, in addition to the research of the standard deviation and the average between improvement classes of the Office Improvement School. It was evident that the standardized grade is currently the most coherent way of comparing different classes and tests, contributing to a more reliable and performance-based performance analysis. Distortions were observed in the grades and averages of the classes of the School of Improvement of Nearby Officers, in which the military compete for the selection processes with non-standard grades, demonstrating a discrepancy of the current system that uses the gross grade. This fact made it possible to design a formula capable of standardizing grades and avoiding distortions in selection processes.

Keywords: Military Schools; Merit; Performance; Evaluation.

Topic: Direito Militar

Reviewed anonymously in the process of blind peer
Received: 22/11/2018

Approved: 23/03/2019
Marcus Vinícius Gomes Pereira

Centro Universitário do Sul de Minas, Brasil http://lattes.cnpq.br/0182704220595324

mgmarcusgomes@gmail.com

Alessandro Messias Moreira (ib

Centro Universitário do Sul de Minas, Brasil

http://lattes.cnpq.br/5303526458310366

http://orcid.org/0000-0001-8120-6219

alessandro.moreira@unis.edu.br

Sheldon William Silva (ii

Instituto Federal do Norte de Minas Gerais, Brasil

http://lattes.cnpq.br/5691436224279198

http://orcid.org/0000-0002-2473-5728

sheldonwilliamsilva@gmail.com
Referencing this:

PEREIRA, M. V. G.; MOREIRA, A. M.; SILVA, S. W.. A influência da avaliação baseada em desempenho no sistema de valorização do mérito: uma proposta para as escolas militares de formação e aperfeiçoamento. Scientiam Juris, v.7, n.1, p.1-10, 2019. DOI: http://doi.org/10.6008/CBPC2318-3039.2019.001.0001 
A influência da avaliação baseada em desempenho no sistema de valorização do mérito: uma proposta para as escolas militares de formação e

\section{INTRODUÇÃO}

Este trabalho se insere na melhoria do sistema de valorização do mérito, utilizado como instrumento nos processos seletivos para cursos, funções, missões no exterior ou qualquer forma de seleção no Exército Brasileiro. A valorização dos recursos humanos no Exército Brasileiro assume uma vertente das mais importantes da instituição, pois sendo predominantemente formada por pessoas, a sua motivação é base para o cumprimento das missões institucionais. A proposta do trabalho é apresentar uma forma de avaliação nas escolas de formação e aperfeiçoamento baseada em desempenho, com notas padronizadas, possibilitando a comparação entre turmas diferentes.

A influência da avaliação baseada em desempenho no sistema de valorização do mérito configura uma ajustagem da forma com que são pontuados critérios da valorização, tendo como problema atual um peso considerável da nota no curso de aperfeiçoamento e sua utilização de forma bruta, possivelmente distorcendo uma avaliação com turmas diferentes de formação e aperfeiçoamento. Tal abordagem se justifica dentro da importância do assunto nos processos seletivos do Exército Brasileiro.

É importante salientar que o atual sistema de valorização utiliza as notas brutas, que possibilita o aparecimento do erro quando o processo seletivo envolve turmas diferentes. Sua contribuição será possibilitar uma avaliação independente da turma, mas de acordo com o desempenho. O objetivo é propor uma forma de cálculo de nota capaz de evitar a distorção existente entre turmas, face às dificuldades das provas em anos diferentes e das formas de avaliação que se modificam conforme as orientações do comando de cada escola. Este propósito será conseguido através de pesquisa junto aos outros estabelecimentos de ensino que utilizam a nota padronizada e através do estudo do reflexo produzido nas turmas que utilizaram a nota bruta.

Atualmente o Sistema de Valorização do Mérito, aprovado pela portaria no 240-DGP, de 23 de outubro de 2013, utiliza as notas brutas obtidas pelos alunos nos cursos de formação e aperfeiçoamento para compor a pontuação da Ficha de Valorização do Mérito (FVM). Cabe ressaltar que todos os processos de promoção e de seleção utilizam a pontuação da Ficha de Valorização do Mérito como um dos critérios para elaboração dos Quadros de Acesso (Processos de Promoção) e dos Mapas de Indicadores (Processos de Seleção). A transformação em pontos dos dados constantes na sua Ficha Cadastro, em conformidade com as Instruções Reguladoras para o Sistema de Valorização do Mérito dos Militares do Exército (EB30-IR-60.001) (BRASIL, 2013a; 2013b).

Considerando que se um dado tem uma avaliação não padronizada, utilizando somente os dados brutos, trazendo, como reflexo, uma valorização assimétrica em relação a outras turmas e, consequentemente, prejuízo ao militar de melhor desempenho, integrante de processo de promoção ou seleção. Cada prova ou avaliação possui critérios que a torna mais difícil ou mais fácil de resolver, refletindo em uma nota bruta que poderá ser maior ou menor, influenciando na média da turma. O sistema atual de valorização do mérito não considera essas diferenças, sendo que os processos seletivos acabam tendo como interessados militares de turmas diferentes, que foram avaliados por critérios diferentes, utilizando somente 
A influência da avaliação baseada em desempenho no sistema de valorização do mérito: uma proposta para as escolas militares de formação e aperfeiçoamento

os dados brutos das suas notas. O problema se intensifica quando considera o curso de aperfeiçoamento que tem sua nota multiplicada por 2 (dois) para oficiais e por 3 (três) para os praças na valorização do mérito, sendo que as turmas não são as mesmas da formação.

O entendimento do desempenho começa a ficar mais complexo a partir do momento em que deixamos de olhar valores numéricos e começamos a olhar outros aspectos do desempenho, considerando o desempenho global, sua média e desvio padrão. O presente trabalho visa apresentar uma forma de avaliação por desempenho e não por notas brutas, realizando uma padronização das notas obtidas pelos militares em cursos e anos diferentes, a qual nos permite identificar desempenhos independentemente da sua turma ou curso realizado.

\section{REVISÃO TEÓRICA}

\section{A avaliação baseada no desempenho e o sistema de valorização do mérito}

Havendo a possibilidade de se ter uma avaliação com diferentes graus de dificuldade e a necessidade de comparar as notas de alunos de turmas diferentes, a padronização da nota se torna necessária para uma avaliação voltada para o desempenho do aluno, possibilitando uma comparação entre turmas diferentes. Ou seja, as notas precisam de um tratamento que minimize as diferenças dos graus de dificuldade do curso naquele ano.

\section{O Sistema de Valorização do Mérito}

O Sistema de Valorização do Mérito é atualmente normatizado pela Portaria no 994, de 18 de dezembro de 2018, tendo como finalidade estabelecer as normas necessárias para o Sistema de Valorização do Mérito dos Militares do Exército, conforme o Artigo 2ำ que expressa:

\footnotetext{
A valorização do mérito tem por objetivos:

I - Orientar os militares de carreira quanto aos componentes da profissão militar considerados prevalentes para a política de pessoal;

II - incentivar o aprimoramento profissional dos integrantes da instituição;

III - ordenar os militares, por mérito, em universo e processo considerado;

IV - constituir um dos critérios de apoio a decisão nos processos seletivos e de promoções conduzidos no âmbito do Exército (Portaria no 994, de 18 de dezembro de 2018, do Comandante do Exército).

Um dos componentes da valorização do mérito, considerado na citada portaria, é a nota em escolas
} de formação e aperfeiçoamento, sendo considerada de grande peso para a valorização. Para a formação, a nota bruta é multiplicada por 1 (um) e no aperfeiçoamento multiplicada por 2 (dois) para os oficiais e por 3 (três) para os praças, podendo perfazer até 30 pontos, um valor considerável para o sistema.

\section{A Padronização de Notas com Base no Desempenho}

A padronização de uma variável já é assunto de estudo da área estatística. De acordo com Silva (1999), o escore z é o quanto uma medida se afasta da média em termos de desvio padrão. Quando o escore z for positivo indica que o dado está acima da média e quando for negativa que o mesmo se encontra abaixo 
da média. Ainda de acordo com o autor, em diversas situações onde temos diversas origens de valores ou escores, é necessário converter os valores brutos em escala padronizada, sendo até uma questão de justiça entre comparações e classificações.

O estudo a respeito do assunto surgiu na inscrição para o teste da Associação Nacional de PósGraduação e Pesquisa em Administração (ANPAD), para fins de inscrição em um Mestrado Profissional em Administração Pública (PROFIAP). $O$ teste da ANPAD tem validade de 2 anos, aplicação média de 3 vezes por ano, então em um processo seletivo que se utiliza a nota da ANPAD, temos 6 turmas diferentes. Assim foi necessário evitar o efeito chamado 'prova' do processo de seleção, realizando assim a padronização das notas.

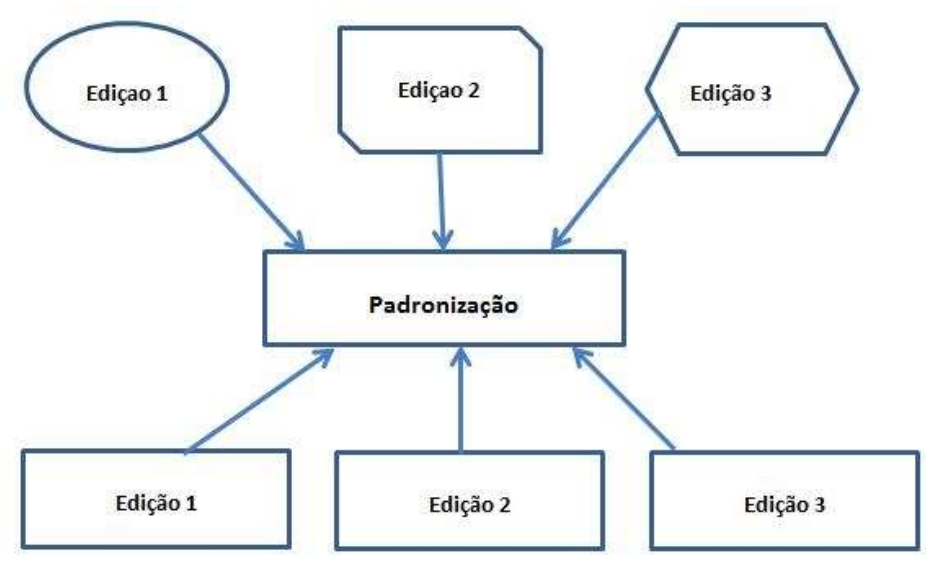

Figura 1: Estrutura de Padronização do Teste ANPAD.

Na padronização da nota do teste da ANPAD são utilizados dois dados, a média das pontuações brutas e o desvio padrão:

Considere-se, por exemplo, o caso de um inscrito que tenha acertado 16 das 20 questões de uma prova. Esse resultado, de $80 \%$, é, à primeira vista, positivo e indicador de bom desempenho do inscrito. No entanto, deve-se ter em mente que, por algum motivo não previsto, a prova pode ter sido relativamente fácil para a população, de modo que 16 foi uma das menores pontuações e é, portanto, indicativo do baixo desempenho do inscrito em relação aos demais. Consequentemente faz-se necessário estabelecer uma base de comparação que leve em conta um indicador do desempenho da população dos inscritos (como, por exemplo, a média da prova) e do grau de homogeneidade das pontuações (como o desvio-padrão).

É importante destacar que, já na fase de elaboração das provas, são seguidos critérios rigorosos para evitar discrepâncias nos níveis de dificuldades do Teste. No entanto, como nem sempre o nível de dificuldade previsto pelos elaboradores e revisores das provas condiz com o nível de dificuldade efetivamente observado na população (por questões como variação na formação dos inscritos), é importante a realização da padronização ora explicada.

Além da ANPAD, a Fundação Carlos Chagas, em processos de concursos públicos, também utiliza critérios de padronização de notas. A padronização da nota de uma disciplina, no vestibular da Fundação Getúlio Vargas, é obtida por meio da seguinte fórmula: NPi $=5+[(\mathrm{Ni}-\mathrm{Mx}) / \mathrm{DPx}]$, na qual; NPi = nota padronizada do candidato i naquela prova; $\mathrm{Ni}$ = nota bruta do candidato $\mathrm{i}$ (antes da padronização) naquela prova; $\mathrm{Mx}=$ média das notas dos candidatos naquela prova, $\mathrm{DPx}=$ desvio-padrão das notas naquela prova. Onde: $\mathrm{NP}=$ Nota Padronizada $\mathrm{N}=$ Nota bruta do candidato $\mathrm{M}=$ Média geral das notas brutas da prova $\mathrm{DP}=$ Desvio Padrão das notas brutas da prova. 
A influência da avaliação baseada em desempenho no sistema de valorização do mérito: uma proposta para as escolas militares de formação e

Conclui-se parcialmente, que as instituições que utilizam as notas em processos seletivos que envolvam turmas diferentes e necessidade de comparação, de alguma forma, padronizam as notas, de forma a comparar o desempenho dos candidatos e não somente a nota bruta. A metodologia de padronização também é adotada pelo College Board, prestigiosa organização sem fins lucrativos, fundada em 1900 com o nome de College Entrance Examination Board (CEEB), sendo responsável pelo processo seletivo para mais de 5.000 escolas americanas.

No Exército, a padronização de nota aumenta de importância, devido sua utilização para o Sistema de Valorização do Mérito e consequentemente, nos processos seletivos de promoção ou designação para cursos, estágio e missões no exterior. A Escola de Sargentos das Armas (ESA) é um estabelecimento de ensino superior (tecnólogo) do Exército responsável pela formação de sargentos combatentes. Exemplificando, se um sargento da ESA da turma de 2004, no seu aperfeiçoamento em 2014 tenha obtido a nota 7,0. Supondo que a nota do curso possa variar de 0,0 a 10,0, em uma escala de proporção, isso significa que acertou $70 \%$ das provas do seu curso. Se a nota mínima para aprovação no curso é 5,0, então esse sargento está aprovado. O entendimento do desempenho começa a ficar mais complexo a partir do momento em que valores numéricos são substituídos por aspectos do desempenho. Por exemplo, compara-se a nota 7,0 com as notas dos demais alunos do curso de aperfeiçoamento de 2014. Essa nota pode ser a melhor do curso, desde que todos os outros alunos tenham tido nota mais baixa; ou a pior, se tiverem tido nota superior a 7,0. Dessa forma, é evidente a necessidade de outras informações, além da nota do aluno; por exemplo, a média de notas da turma.

Se essa média tenha sido 6,25, essa informação permite concluir que o desempenho do aluno foi superior à média da turma. Agora se outro sargento da mesma turma de formação (2004) tenha obtido no seu curso de aperfeiçoamento, que foi realizado em 2015, também, nota 7,0. Isso significa que 70\% das notas provas do seu curso estão corretas e ele obteve nota maior que a mínima para aprovação, se comparar a sua nota no curso com a média da turma, sendo que esta tenha sido 5,0, nesse caso, o desempenho dele foi superior ao da média da turma. Se os dois sargentos obtiveram nota 7,0 (70\% de acertos) no Curso de Aperfeiçoamento, podemos afirmar que o desempenho deles é igual? Para responder a essa pergunta, considera-se outro fator importante: a variabilidade de notas dos alunos. Ilustra-se no exemplo na tabela 1.

Tabela 1: Média de Notas das Turmas de 2014 e 2015.

\begin{tabular}{|c|c|c|c|c|c|}
\hline \multicolumn{3}{|c|}{ TURMA 2014} & \multicolumn{3}{|c|}{ TURMA 2015} \\
\hline ALUNO & NOTA & MÉDIA & ALUNO & NOTA & MÉDIA \\
\hline$A$ & 4,8 & \multirow{6}{*}{6,25} & $\mathrm{~A}$ & 2,0 & \multirow{6}{*}{5,0} \\
\hline$B$ & 5,5 & & $\mathrm{~B}$ & 3,0 & \\
\hline $\mathrm{C}$ & 6,5 & & $\mathrm{C}$ & 4,0 & \\
\hline $\mathrm{D}$ & 6,5 & & $\mathrm{D}$ & 6,0 & \\
\hline$E$ & 7,0 & & $\mathrm{E}$ & 7,0 & \\
\hline $\mathrm{F}$ & 7,2 & & $\mathrm{~F}$ & 8,0 & \\
\hline
\end{tabular}

Visualmente percebe-se que a dispersão das notas do aperfeiçoamento 2014 (mínima 2,0 e máxima 8,0) foi maior que a dispersão das notas do aperfeiçoamento 2015 (mínima 4,8 e máxima 7,2). Uma medida de dispersão dos elementos que compõem a média é o desvio-padrão. É um número que mede em quanto 
A influência da avaliação baseada em desempenho no sistema de valorização do mérito: uma proposta para as escolas militares de formação e

os elementos estão menos ou mais dispersos em relação à média. Considerando o exemplo acima, o desviopadrão das notas do CAS 2014 é 0,84 enquanto o desvio-padrão do CAS 2015 é 2,16. Quando examina-se o desempenho, uma série de cuidados deverão ser tomados, considerando as características desse tipo de avaliação, pois o cálculo considera o melhor desempenho. O melhor desempenho não pode ser aferido por meio de notas absolutas, pois, conforme visto acima, o desempenho deve ser comparado com a média dos outros candidatos e também com as medidas de dispersão dos elementos que compõem essa média.

Exemplo de cálculo simples, um sargento que tenha obtido nota 7,0 dentre o seguinte grupo: Turma de 6 alunos (notas): 5,6-5,7-5,8-5,9-6,0-7,0 (repare que a nota média foi 6,0). Nesse caso, o desviopadrão das notas brutas é igual a 0,465. A nota padronizada desse aluno será: NP $=5+(7-6) / 0,47=7,15$. A sistemática de padronização de notas é uma metodologia que traz uma forma justa de quantificar valores considerando os melhores desempenhos, independente do ano do curso, pois considera o desempenho global dos alunos.

\section{Os Reflexos no Sistema de Valorização do Mérito}

A utilização da nota bruta no sistema de valorização do mérito vem causando distorções e possivelmente prejuízos, primeiramente para o Exército que não está selecionando o mais capaz, e também prejuízo para o militar que integrou uma turma com média baixa na formação ou aperfeiçoamento. É fácil verificar essa distorção quando analisamos os dados e as notas dos últimos anos do Curso de Aperfeiçoamento de Oficiais, vamos utilizar a Curva de Gauss das notas entre 2009 e 2018, conforme figura 2. Ao analisar melhor um único curso, suas notas entre 2009 até 2015, estabelecidas na tabela 2.

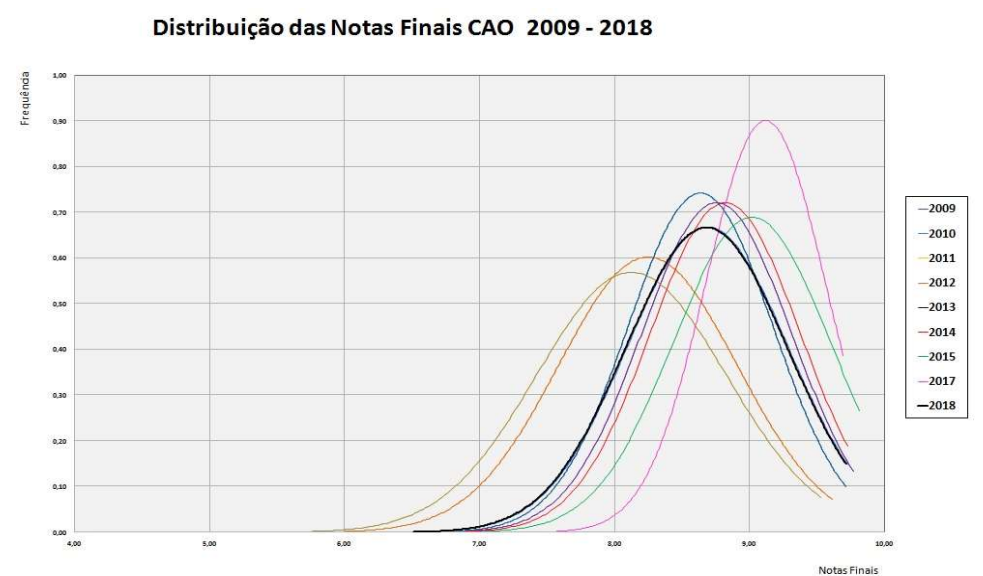

Figura 2: Distribuição de Notas Finais do Curso de Aperfeiçoamento de Oficiais 2009-2018.

É possível verificar que a turma de 2011 teve prejuízos por conta do efeito 'curso', onde as provas e a forma de cálculo da nota naquele ano levaram a uma nota baixa em relação aos demais anos. Neste ensejo, posteriormente a nota foi multiplicada por 2 (dois) e incorporada ao sistema de valorização do mérito, causando prejuízo em processos seletivos e promoções dos militares que realizaram o curso de aperfeiçoamento em 2011. 
A influência da avaliação baseada em desempenho no sistema de valorização do mérito: uma proposta para as escolas militares de formação e aperfeiçoamento

PEREIRA, M. V. G.; MOREIRA, A. M.; SILVA, S. W.

Tabela 2: Dados do Curso de Intendência da Escola de Aperfeiçoamento de Oficiais.

\begin{tabular}{|l|l|l|l|l|l|l|l|}
\hline \multirow{2}{*}{ Dado Estatístico } & \multicolumn{5}{l|}{ CURSO DE INTENDÊNCIA } \\
\cline { 2 - 9 } & 2009 & 2010 & 2011 & 2012 & 2013 & 2014 & 2015 \\
\hline Desvio Padrão & 0,521 & 0,464 & 0,625 & 0,772 & 0,601 & 0,806 & 0,484 \\
\hline Média & 9,017 & 8,938 & 7,825 & 7,923 & 8,314 & 8,929 & 9,297 \\
\hline Mediana & 9,155 & 9,024 & 7,991 & 8,059 & 8,199 & 9,144 & 9,417 \\
\hline Maior & 9,761 & 9,616 & 8,755 & 9,244 & 9,305 & 9,712 & 9,778 \\
\hline Menor & 6,856 & 6,788 & 5,918 & 5,503 & 7,044 & 5,090 & 7,365 \\
\hline
\end{tabular}

Transformando em números essa constatação, basta verificar que o 1ำ colocado em 2011 obteve nota de 8,7 gerando 17,4 pontos na quantificação do mérito, o primeiro colocado de 2010 obteve a nota bruta de 9,6 gerando 19,2 pontos na quantificação do mérito, sendo que os dois militares são da mesma turma de formação, participam do mesmo processo de promoção e possivelmente de seleção para outros casos, mas o militar que cursou a Escola de Aperfeiçoamento de Oficiais em 2010 tem quase dois pontos de vantagem. Ficando claro o efeito 'ano do curso' neste caso.

\section{MATERIAIS E MÉTODOS}

O objetivo principal dessa pesquisa é demonstrar a necessidade de realizar uma avaliação padronizada, baseada no desempenho do aluno para possibilitar a seleção de militares de diferentes turmas de formação e aperfeiçoamento. Como propósito de estudo, busca-se encontrar uma fórmula baseada na média e no desvio padrão, capaz de padronizar as notas dos alunos e possibilitar a comparação entre turmas diferentes.

A pesquisa pode ser considerada como descritiva. Gil (2002) "as pesquisas descritivas têm como objetivo primordial a descrição das características de determinada população ou fenômeno ou, então, o estabelecimento de relações entre variáveis". A presente pesquisa é bibliográfica e documental emprega fundamentalmente contribuições de diversos autores sobre determinado assunto e por meio de um comparativo com outras instituições que já utilizam a nota padronizada, além da pesquisa do desvio padrão e da média entre turmas de aperfeiçoamento da Escola de Aperfeiçoamento de Oficiais (GIL, 2002).

O universo utilizado na pesquisa foi composto das turmas de intendência entre os anos de 2009 a 2015, apenas para materializar dados e projetar os reflexos no sistema de valorização do mérito. Com as notas obtidas na ESAO, foi possível projetar a curva de Gauss, necessária para uma avaliação inicial da distribuição das notas em anos diferentes. De acordo com Bittencourt et al. (2006), a distribuição normal ou curva de Gauss-Moivre-Laplace é o principal modelo probabilístico contínuo, pois serve de base para a principal área da Estatística: a Inferência. A distribuição normal ou curva de Gauss é um modelo que descreve o comportamento de vários fenômenos aleatórios.

A pesquisa realizada assume ainda características quantitativas e qualitativas que respectivamente pretendem: (i) realizar cálculos matemáticos e estatísticos para obter as discrepâncias e também buscar a solução para o problema; (ii) compreender as causas e efeitos da utilização da nota bruta nos processos seletivos do Exército. 
A influência da avaliação baseada em desempenho no sistema de valorização do mérito: uma proposta para as escolas militares de formação e

\section{RESULTADOS E DISCUSSÃO}

Ao realizar a revisão bibliográfica sobre o assunto, nota-se que diversos processos seletivos que utilizam a forma atemporal de aplicação de prova, têm a padronização da nota como uma ferramenta fundamental para comparar resultados. Através da constatação da discrepância e a análise com base na revisão bibliográfica do assunto, possibilitou trabalhar em uma fórmula capaz de evitar a análise com erro e obter uma comparação dos dados com base no desempenho de cada aluno. Ao relacionar as notas com o sistema de valorização do mérito, é possível visualizar as diferenças e algumas discrepâncias que possivelmente prejudicaram militares em processos seletivos ou nas promoções.

A proposta apresentada para o Exército Brasileiro utilizar nos Curso de Formação e Aperfeiçoamento, segue a seguinte didática: (a) é considerada a nota bruta obtida no final do curso de cada militar; (b) são calculadas a média e o desvio padrão dos acertos de todos os militares da mesma turma. Média: é a soma das notas brutas de todos os alunos dividida pelo total de alunos. Desvio padrão: é a raiz quadrada da variância. (Variância é a média aritmética dos quadrados dos desvios). O desvio padrão é uma medida de dispersão, que mede o quanto os dados se distanciam da média. Para efeitos didáticos, consideramos que uma turma de aperfeiçoamento de sargentos de 2010 tenha o efetivo de 05 militares (A-B-C-D-E). As notas brutas ao término do curso estão na tabela 3.

Tabela 3: Notas por Aluno.

\begin{tabular}{|l|l|}
\hline ALUNO & NOTA \\
\hline A & 9,8 \\
\hline B & 7,7 \\
\hline C & 7,9 \\
\hline D & 8,5 \\
\hline E & 8,8 \\
\hline
\end{tabular}

Determinando a média e o desvio padrão dos acertos de todos os alunos que realizaram o curso:

Desvio Padrão $=0,8324$

Média $=8,54$

Fórmula 01:

Nota Padronizada (NP) $=\underline{\text { Nota Bruta (NB) }- \text { Média }}+8$ Desvio Padrão (DP)

Tabela 4: Cálculo da Nota Padronizada.

\begin{tabular}{|l|l|l|l|}
\hline ALUNO & NOTA BRUTA & \multirow{2}{*}{ MÉDIA } & NOTA PADRONIZADA \\
\cline { 1 - 1 } A & $\mathbf{8}, \mathbf{5 4}$ & 9,51 \\
\hline B & & & 7,00 \\
\hline C & 7,7 & DESVIO PADRÃO & 7,23 \\
\hline$D$ & 7,9 & $\mathbf{0} 8324$ & 8,45 \\
\hline E & 8,5 & & 8,49 \\
\hline
\end{tabular}

Outro exemplo: Agora a turma de aperfeiçoamento de 2011, que foi mais exigida e por consequência obteve uma média mais baixa, possuindo também 05 militares (A-B-C-D-E). As notas brutas ao término do curso estão na tabela 5 .

Tabela 5: Notas por Aluno.

\begin{tabular}{|l|l|}
\hline ALUNO & NOTA \\
\hline A & 8,8 \\
\hline B & 8,2 \\
\hline
\end{tabular}




\begin{tabular}{|l|l|}
\hline$C$ & 7,6 \\
\hline$D$ & 7,9 \\
\hline$E$ & 8,0 \\
\hline
\end{tabular}

Desvio Padrão $=0,45$

Média $=8,10$

Retomando a fórmula 01:

Nota Padronizada (NP) $=$ Nota Bruta (NB) - Média +8

Desvio Padrão (DP)

Tabela 6: Cálculo da Nota Padronizada - Exemplo 2.

\begin{tabular}{|l|l|l|l|}
\hline ALUNO & NOTA BRUTA & MÉDIA & NOTA PADRONIZADA \\
A & $\mathbf{8 , 1 0}$ & 9,55 \\
\hline B & 8,8 & & 8,22 \\
\hline C & 8,2 & DESVIO PADRÃO & 6,88 \\
\hline D & 7,6 & $\mathbf{0 , 4 5}$ & 7,55 \\
\hline$E$ & 7,9 & & 7,78 \\
\hline
\end{tabular}

Com as notas padronizadas é possível comparar as turmas de 2010 e 2011 para os mesmos processos seletivos, sem o prejuízo do efeito 'ano do curso'.

\section{CONCLUSÕES}

Nesta fase do artigo é importante retomar a curva de Gauss apresentada no desenvolvimento, onde fica evidente que nenhuma turma de aperfeiçoamento teve uma padronização de notas ou manteve o mesmo nível de exigência. Cada ano teve uma curva de notas diferente de qualquer outro ano, com médias e desvios também diferentes, concluindo que a utilização das notas brutas é inadequada para um processo de seleção com turmas diferentes. Também durante a pesquisa do artigo é possível perceber a preocupação de instituições importantes de ensino com a padronização das notas, evitando o efeito 'prova' em seus processos seletivos.

É oportuno retomar o objetivo do artigo de apresentar um cálculo padronizado do grau final obtido nas escolas de formação e aperfeiçoamento que integram o Sistema de Valorização do Mérito de forma a evitar discrepâncias entre as turmas e obter uma avaliação baseada no desempenho. Ainda durante a pesquisa foi possível constatar a referida discrepância e sua influência no sistema de valorização do mérito. Para o cálculo da nota padronizada foi apresentada a seguinte fórmula 1.

Fórmula 1: Padronização de Nota

$$
\text { Nota Padronizada }(N P)=\frac{\text { Nota Bruta }(N B)-\text { Média }}{\text { Desvio Padrão (DP) }}+8
$$

Conclui-se que o sistema atual gera discrepâncias e possibilita a existência de prejuízos nos processos seletivos e ainda que a aplicação da fórmula de padronização de nota irá evitar tais prejuízos e possibilitará uma avaliação voltada para o desempenho de cada militar, independente da sua turma de formação ou aperfeiçoamento.

Este artigo requer um maior aprofundamento no grau de impacto das notas dos cursos de formação e aperfeiçoamento no sistema de valorização do mérito a fim de materializar em valores sua representatividade no referido sistema, em uma visão parcial se percebe uma significativa importância. Ainda 
exige um estudo na forma de aplicação da mudança, onde seria realizada, na própria escola ou quando da sua inserção no sistema de valorização, se atingirá somente as turmas atuais e futuras ou com efeito retroativo.

\section{REFERÊNCIAS}

BITTENCOURT, H. R.; VIALI, L.. Contribuições para o ensino da distribuição normal ou curva de Gauss em cursos de graduação. In: SEMINÁRIO INTERNACIONAL DE PESQUISA EM EDUCAÇÃO MATEMÁTICA. Anais. SIPEM, 2006.

BRASIL. Exército Brasileiro. EB30-IR-60.001: Instruções Reguladoras para o Sistema de Valorização do Mérito. Brasília: Exército Brasileiro, 2013a.
BRASIL. Exército Brasileiro. Portaria n.240-DGP: Valorização do Mérito. Brasília: Exército Brasileiro, 2013b.

GIL, A. C.. Como elaborar projetos de pesquisa. São Paulo: Atlas, 2002.

SILVA, P. A. L.. Probabilidade e Estatística. Rio de Janeiro: Reichmann \& Affonso, 1999.

A CBPC - Companhia Brasileira de Produção Científica (CNPJ: 11.221.422/0001-03) detém os direitos materiais desta publicação. Os direitos referem-se à publicação do trabalho em qualquer parte do mundo, incluindo os direitos às renovações, expansões e disseminações da contribuição, bem como outros direitos subsidiários. Todos os trabalhos publicados eletronicamente poderão posteriormente ser publicados em coletâneas impressas sob coordenação da Sustenere Publishing, da Companhia Brasileira de Produção Científica e seus parceiros autorizados. Os (as) autores (as) preservam os direitos autorais, mas não têm permissão para a publicação da contribuição em outro meio, impresso ou digital, em português ou em tradução. 